\title{
THE MODULI OF COMPACT CONTINUATIONS OF AN OPEN RIEMANN SURFACE OF GENUS ONE
}

\author{
M. SHIBA \\ Dedicated to Professor Isamu Tsukahara on his 65th birthday
}

\begin{abstract}
Let $(R,\{A, B\})$ be a marked open Riemann surface of genus one. Denote by $\left(T,\left\{A_{T}, B_{T}\right\}, i\right)$ a pair of a marked torus $\left(T,\left\{A_{T}, B_{T}\right\}\right)$ and a conformal embedding $i$ of $R$ into $T$ with $i(A)$ and $i(B)$ homotopic respectively to $A_{T}$ and $B_{T}$. We say that $\left(T,\left\{A_{T}, B_{T}\right\}, i\right)$ and $\left(T^{\prime},\left\{A_{T^{\prime}}, B_{T^{\prime}}\right\}, i^{\prime}\right)$ are equivalent if $i^{\prime} \circ i^{-1}$ extends to a conformal mapping of $T$ onto $T^{\prime}$. The equivalence classes are called compact continuations of $(R,\{A, B\})$ and the set of moduli of compact continuations of $(R,\{A, B\})$ is denoted by $M=M(R,\{A, B\})$. Then $M$ is a closed disk in the upper half plane. The radius of $M$ represents the size of the ideal boundary of $R$ and gives a generalization of Schiffer's span for planar domains; in particular, it vanishes if and only if $R$ belongs to the class $O_{A D}$. On the other hand, any holomorphic differential on $R$ with distinguished imaginary part produces in a canonical manner a compact continuation of $(R,\{A, B\})$. Such a compact continuation is referred to as a hydrodynamic continuation of $(R,\{A, B\})$. The boundary of $M$ parametrizes in a natural way the space of hydrodynamic continuations; i.e., the hydrodynamic continuations have extremal properties.
\end{abstract}

Introduction. A Riemann surface $R_{0}$ is called a continuation (or prolongation, extension) of another Riemann surface $R$, if there exists a conformal injection of $R$ into $R_{0}$. See Bochner $[\mathbf{3}]$ and Sario-Oikawa $[\mathbf{2 2}]$, for example. We are specifically interested in the case where $R$ is an open Riemann surface of finite genus and $R_{0}$ is a closed Riemann surface of the same genus. The existence of such $R_{0}$ (for a given $R$ ) is a classical result, and the totality of those $R_{0}$ 's for a fixed $R$ is also studied by many authors in the framework of Teichmüller space theory. Cf. Heins [9], Ioffe $[\mathbf{1 1}, \mathbf{1 2}]$, Oikawa $[\mathbf{1 5}, \mathbf{1 6}]$, Renggli $[\mathbf{1 9}]$, and Timmann $[\mathbf{3 1}]$.

In the present paper we shall restrict ourselves, as in [9], only to the case of genus one. Then, we obtain a refinement of Heins' result; we give a complete description of the moduli set of compact continuations of a fixed open Riemann surface of genus one. (In [9] Heins considered the moduli space rather than the Teichmüller space. But here we are concerned with the latter.)

Received by the editors March 11, 1986.

1980 Mathematics Subject Classification (1985 Revision). Primary 30Fxx, 32G15; Secondary 14H15, 30C75, 76B99.

Key words and phrases. Continuation (prolongation, extension) of Riemann surfaces, modulus (of a torus), Teichmüller space, extremal slit torus, span, $O_{A D}$, hydrodynamic continuation, complex velocity potential, Strömungsfunktion, distinguished harmonic differential, principal function.

The author thanks Professor K. Oikawa at the University of Tokyo for stimulating conversations and advice. He also expresses his hearty gratitude to Professor J. A. Jenkins at Washington University who brought his attention to Grötzsch's paper [6] via Professor Oikawa. The referee and Dr. H. Köditz at the University of Hannover pointed out some improper versions in the first draft. The author thanks them for their careful reading and kindness. 
To explain our results, let $R$ be an open Riemann surface of genus one with a fixed canonical homology basis $\{A, B\}$ modulo dividing cycles (cf. [2]). (We may assume that $A \cap B$ consists of a single point.) The system $\{A, B\}$ is also a set of generators for the fundamental group of the Kerékjártó-Stoilow compactification of $R$ (cf. [20]). Hence, following the standard terminology for compact surfaces, we shall call the pair $(R,\{A, B\})$ a marked open Riemann surface.

By a marked realization of $(R,\{A, B\})$ we mean a triple $\left(T,\left\{A_{T}, B_{T}\right\}, i\right)$, where $\left(T,\left\{A_{T}, B_{T}\right\}\right)$ is a marked torus in the ordinary sense and $i$ is a conformal embedding of $R$ into $T$ with $i(A)$ and $i(B)$ homotopic respectively to $A_{T}$ and $B_{T}$. Two marked realizations $\left(T,\left\{A_{T}, B_{T}\right\}, i\right)$ and $\left(T^{\prime},\left\{A_{T^{\prime}}, B_{T^{\prime}}\right\}, i^{\prime}\right)$ are said to be equivalent if $i^{\prime} \circ i^{-1}$ extends to a conformal mapping of $T$ onto $T^{\prime}$. Each equivalence class is called a compact continuation of $(R,\{A, B\})$. Since equivalent marked realizations have the same modulus (with respect to their markings), we can speak of the modulus of a compact continuation. We denote by $M(R,\{A, B\})$ the set of moduli of compact continuations of $(R,\{A, B\})$.

For any $t,-1<t \leq 1$, there exists a unique holomorphic differential $\phi_{t}$ on $R$ such that $\operatorname{Im}\left[e^{-\frac{1}{2} \pi i t} \phi_{t}\right]$ is distinguished $[2]$ and $\int_{A} \phi_{t}=1$. Furthermore, we know [26] the existence of a marked realization $\left(T_{t},\left\{A_{t}, B_{t}\right\}, i_{t}\right)$ of $(R,\{A, B\})$ such that the transplant of $\phi_{t}$ by $i_{t}^{-1}$ extends to the normal holomorphic differential $\phi^{T_{t}}$ on $T_{t}$ with respect to the basis $\left\{A_{t}, B_{t}\right\}$. The compact continuation represented by $\left(T_{t},\left\{A_{t}, B_{t}\right\}, i_{t}\right)$ will be referred to as the hydrodynamic continuation of $(R,\{A, B\})$ with respect to $\phi_{t}$, since $\phi_{t}$ is associated with a stationary flow of an ideal fluid on $R$. The ideal boundary of $R$ is then realized on $T_{t}$ (via $i_{t}$ ) as a set of arcs on the streamlines of the flow with which $\phi^{T_{t}}$ is associated. (We may always assume $A_{t}$ is geodesic with respect to the metric $\left|\phi^{T_{t}}\right|$. Then $i_{t}(R)$ is a torus with geodesic parallel slits of inclination $\frac{1}{2} \pi t$ with $A_{t}$. The total area of these slits is equal to zero.)

Now our results are as follows.

(I) $M(R,\{A, B\})$ is a closed disk in the Teichmüller space of genus one (the upper half plane).

(II) To each boundary point of $M(R,\{A, B\})$ there corresponds a unique hydrodynamic continuation of $(R,\{A, B\})$, and vice versa.

(III) To an interior point of $M(R,\{A, B\})$ there correspond in general more than one compact continuation of $(R,\{A, B\})$.

(IV) The radius $\rho(R)$ of $M(R,\{A, B\})$ vanishes if and only if $R \in O_{A D}$. The quantity $\sigma(R):=\rho(R) / \pi$ gives a generalization of Schiffer's span for planar regions.

In the proof of (I)-(IV), our preceding results $[\mathbf{2 6}, \mathbf{2 7}]$ will be very useful. Similar results had been obtained by Grötzsch $[6]$. Our method is completely different from his, however.

\section{Preliminaries.}

1.1. Along the line of Riemann, Klein and Hilbert, the class of single-valued meromorphic functions which describe stationary dipole flows of an ideal fluid on a planar surface has played an important role in the theory of conformal mapping. Each function in this class is referred to as a "Strömungsfunktion" (see [10]) or, for short, an "S-function" [26]. In fluid dynamics it is usually called a complex velocity potential. Such a function furnishes a one-to-one conformal mapping of the surface 
considered onto a so-called (extremal) parallel slit region. (Cf. $[\mathbf{2}, \mathbf{1 0}, \mathbf{2 1}, \mathbf{2 2}$, and 29].)

In $[\mathbf{2 6}, \mathbf{2 7}]$, we have been concerned with a generalized "Strömungsfunktion"; it describes a general multipole stationary flow on a surface of positive finite genus and is not necessarily single-valued. Indeed, the important thing for physical phenomena is the (single-valued) derivative with respect to any fixed local parametermore precisely the differential-rather than the (multiple-valued) complex velocity potential itself. Even if the complex velocity potential is not single-valued, it still determines a uniform ("einförmig" in the sense of Klein) flow on the surface. Therefore, in the present paper too, we shall understand the term "S-function" in this wider sense.

Now we shall give a formal definition of an S-function in a modern terminology. Let $R$ be an open Riemann surface, $f$ a single- or multiple-valued meromorphic function on $R$, and $t$ a real number, $-1<t \leq 1$. We call $f$ an $\mathrm{S}_{t}$-function if $\operatorname{Im}\left[e^{-\frac{1}{2} \pi i t} d f\right]$ is a distinguished harmonic differential of Ahlfors (see [2, p. 313]). When it is not necessary to refer to $t$ explicitly, we use the term $S$-function.

If $f$ is an $\mathrm{S}_{t}$-function, then $e^{-\frac{1}{2} \pi i(t+1)} d f$ is a canonical semiexact differential of Kusunoki, and vice versa. In case that $\operatorname{Im}\left[e^{-\frac{1}{2} \pi i t} f\right]$ (resp. $\operatorname{Re}\left[e^{-\frac{1}{2} \pi i t} f\right]$ ) is singlevalued on the whole surface, $f$ is an $\mathrm{S}_{t}$-function if and only if $\operatorname{Im}\left[e^{-\frac{1}{2} \pi i t} f\right]$ (resp. $\left.\operatorname{Re}\left[e^{-\frac{1}{2} \pi i t} f\right]\right)$ is a $(Q) L_{1^{-}}\left(\right.$resp. $\left.L_{0^{-}}\right)$principal function of Sario. See [21 or 22].

The present work heavily depends on the following theorem $[\mathbf{2 6}, \mathbf{2 7}]$ :

THEOREM 1. Let $R$ be an open Riemann surface of finite genus $g$ and $\Phi$ an $S_{t}$ function on $R,-1<t \leq 1$. Then there is a closed Riemann surface $R^{\Phi}$ of genus $g$, a conformal embedding $i: R \rightarrow R^{\Phi}$, and a single- or multiple-valued meromorphic function $\tilde{\Phi}$ on $R^{\Phi}$ such that

(1) $R^{\Phi} \backslash i(R)$ is of zero area,

(2) $i^{*}(d \tilde{\Phi})=d \Phi, i^{*}(d \tilde{\Phi})$ being the pull-back of $d \tilde{\Phi}$ via $i$,

(3) every component of $R^{\Phi} \backslash i(R)$ is a possibly branched arc (or a single point) on the trajectories of the quadratic differential $e^{-\pi i t}(d \tilde{\Phi})^{2}$, and

(4) $\tilde{\Phi}$ is single-valued if and only if $\Phi$ is.

REMARK. $R^{\Phi}$ is not always uniquely determined by $R$ and $\Phi$, but depends on $(2 g-2-d)$-real parameters, where $d$ is the degree of the divisor of $d \Phi$. Cf. [27].

1.2. In this paper we shall deal with the case $g=1$. By Theorem 1 and the above remark, we have then

THEOREM $1^{\prime}$. Let $\Phi$ be a regular $S$-function on an open Riemann surface $R$ of genus one. Then there exists a conformal injection $i$ of $R$ into a torus $T$ such that the transplant of $d \Phi$ by $i^{-1}$ extends to a holomorphic differential on $T$. If $j$ is another conformal injection of $R$ into a torus $S$ such that the transplant of $d \Phi$ by $j^{-1}$ extends to a holomorphic differential on $S$, then there is a conformal mapping $f$ of $T$ onto $S$ with $f \circ i=j($ on $R)$.

Now, fix a canonical homology basis $\{A, B\}$ of $R$ modulo dividing cycles. (We may assume that $A \cap B$ consists of a single point.) We may regard $\{A, B\}$ as a set of generators for the fundamental group of the Kerékjártó-Stoilow compactification of $R$ (see Richards [20]): The pair $(R,\{A, B\})$ will be thus called a marked open Riemann surface. 
Let $\left(T,\left\{A_{T}, B_{T}\right\}\right)$ be a marked torus in the ordinary sense; $T$ shall be a torus and $\left\{A_{T}, B_{T}\right\}$ a set of generators for the fundamental group of $T$. We may assume, without loss of generality, that $A_{T}$ and $B_{T}$ are geodesics with respect to the metric $|d w|$, where $d w$ is an arbitrarily fixed holomorphic differential on $T$. Specifically, we can take $d w$ as the normal differential of the first kind with respect to $\left\{A_{T}, B_{T}\right\}$ : $\int_{A_{T}} d w=1$. If we set

$$
\tau=\tau\left(T,\left\{A_{T}, B_{T}\right\}\right)=\int_{B_{T}} d w,
$$

then $\tau$ is the modulus of $T$ with respect to the marking $\left\{A_{T}, B_{T}\right\}$ and $\operatorname{Im} \tau$ is, as is well known, always positive.

If there exists a conformal injection $i$ of $R$ into $T$ such that $i(A)$ and $i(B)$ are homotopic respectively to $A_{T}$ and $B_{T}$, we call the triple $\left(T,\left\{A_{T}, B_{T}\right\}, i\right)$ a marked realization of $(R,\{A, B\})$. Two marked realizations $\left(T,\left\{A_{T}, B_{T}\right\}, i\right)$ and $\left(T^{\prime},\left\{A_{T^{\prime}}, B_{T^{\prime}}\right\}, i^{\prime}\right)$ of the same $(R,\{A, B\})$ are defined to be equivalent, if there exists a conformal mapping $f$ of $T$ onto $T^{\prime}$ with $f \circ i=i^{\prime}$. Each equivalence class will be called a compact continuation of $(R,\{A, B\})$, and the compact continuation represented by a marked realization $\left(T,\left\{A_{T}, B_{T}\right\}, i\right)$ will be denoted by $\left[T,\left\{A_{T}, B_{T}\right\}, i\right]$. We denote by $C(R,\{A, B\})$ the space of all compact continuations of $(R,\{A, B\})$.

Since equivalent marked realizations obviously define the same point in the Teichmüller space of genus one and so have the same modulus, we can speak of the modulus of a compact continuation. We set $\tau\left[T,\left\{A_{T}, B_{T}\right\}, i\right]=\tau\left(T,\left\{A_{T}, B_{T}\right\}\right)$ for $\left[T,\left\{A_{T}, B_{T}\right\}, i\right] \in C(R,\{A, B\})$ and denote by $M(R,\{A, B\})$ the set of those $\tau\left[T,\left\{A_{T}, B_{T}\right\}, i\right],\left[T,\left\{A_{T}, B_{T}\right\}, i\right] \in C(R,\{A, B\})$. In [9] Heins proved that $M(R,\{A, B\})$ is a compact set. One of our aims is to prove that it is a closed disk in the upper half plane.

As we shall see later, for each $t \in(-1,1]$ there is a unique holomorphic differential $\phi_{t}=d \Phi_{t}$ such that $\Phi_{t}$ is an $\mathrm{S}_{t}$-function and $\int_{A} d \Phi_{t}=1$. Furthermore, by Theorem $1^{\prime}$, there is a torus $T_{t}$ and a conformal embedding $i_{t}$ of $R$ into $T_{t}$ such that the transplant of $d \Phi_{t}$ by $i_{t}^{-1}$ extends to a holomorphic differential $\phi^{T_{t}}$ on $T_{t}$. It is obvious that $\left\{i_{t}(A), i_{t}(B)\right\}$ is a set of generators for the fundamental group of $T_{t}$. Let $A_{t}=A_{T_{t}}$ and $B_{t}=B_{T_{t}}$ be geodesics (with respect to the metric $\left|\phi^{T_{t}}\right|$ ) homotopic respectively to $i_{t}(A)$ and $i_{t}(B)$. Then $\left(T_{t},\left\{A_{t}, B_{t}\right\}, i_{t}\right)$ yields a marked realization of $(R,\{A, B\})$. Using Theorem 1 , we know that $T_{t} \backslash i_{t}(R)$ is a null set whose connected components are geodesic parallel slits of inclination $\frac{1}{2} \pi t$ with $A_{t}$. It is not difficult to see that any marked realization of $(R,\{A, B\})$ equivalent to $\left(T_{t},\left\{A_{t}, B_{t}\right\}, i_{t}\right)$ has the same properties. The compact continuation that is represented by $\left(T_{t},\left\{A_{t}, B_{t}\right\}, i_{t}\right)$ is referred to as the hydrodynamic continuation of $(R,\{A, B\})$ with respect to $d \Phi_{t}$. Its modulus is given by $\int_{B_{t}} \phi^{T_{t}}=\int_{B} d \Phi_{t}$. We shall prove later that the set of moduli of hydrodynamic continuations of $(R,\{A, B\})$ is precisely the boundary of $M(R,\{A, B\})$.

2. Some elementary lemmas. Let $(R,\{A, B\})$ be a marked open Riemann surface of genus one. In this section we shall state some lemmas.

LEMMA 1. For every $t \in(-1,1]$ there exist holomorphic differentials $\phi_{t}^{A}$ and $\phi_{t}^{B}$ such that

(i) $\operatorname{Re}\left[e^{-\frac{1}{2} \pi i t} \phi_{t}^{A}\right]$ and $\operatorname{Re}\left[e^{-\frac{1}{2} \pi i t} \phi_{t}^{B}\right]$ are distinguished harmonic differentials, and 
(ii)

$$
\begin{array}{lll}
\int_{A} \phi_{t}^{A}=i a_{t}^{\prime}, & & \int_{B} \phi_{t}^{A}=-1+i a_{t}^{\prime \prime}, \\
\int_{A} \phi_{t}^{B}=1+i b_{t}^{\prime}, & & \int_{B} \phi_{t}^{B}=i b_{t}^{\prime \prime}
\end{array}
$$

for real numbers $a_{t}^{\prime}, a_{t}^{\prime \prime}, b_{t}^{\prime}$ and $b_{t}^{\prime \prime}$.

Such differentials $\phi_{t}^{A}$ and $\phi_{t}^{B}$ (and hence the numbers $a_{t}^{\prime}, a_{t}^{\prime \prime}, b_{t}^{\prime}$ and $b_{t}^{\prime \prime}$ ) are uniquely determined by $\{A, B\}$ and $t$.

This lemma was established in [25].

The following lemma is a generalization of the famous bilinear relation due to Legendre and Riemann. For the proof, see [25].

LEMMA 2. Let $\phi, \psi$ be holomorphic differentials on $R$ whose imaginary parts are both distinguished. Then

$$
\begin{gathered}
\operatorname{Im}\left(\int_{A} \phi \int_{B} \psi-\int_{B} \phi \int_{A} \psi\right)=0 \\
-2 \operatorname{Im}\left(\int_{A} \phi \int_{B} \bar{\phi}\right)=\|\phi\|_{R}^{2} \geq 0
\end{gathered}
$$

where $\|\phi\|_{R}=\left(\iint_{R} \phi \wedge \bar{\phi}^{*}\right)^{1 / 2}$ is the Dirichlet norm of $\phi$.

The same holds for holomorphic differentials $\phi, \psi$ whose real parts are both distinguished.

From the above lemma easily follows the next lemma.

LEMMA 3. For every $t \in(-1,1]$

(i) $a_{t}^{\prime \prime}=b_{t}^{\prime}$, and

(ii) $a_{t}^{\prime}>0, b_{t}^{\prime \prime}>0$.

LEMMA 4. For every $t \in(-1,1]$ there exists a unique holomorphic differential $\phi_{t}$ on $R$ such that

(i) $\operatorname{Im}\left[e^{-\frac{1}{2} \pi i t} \phi_{t}\right]$ is distinguished, and

(ii) $\int_{A} \phi_{t}=1$.

ProOF. By Lemma $3, \alpha:=\int_{A} \phi_{t}^{A}$ is a nonvanishing purely imaginary number. Then $\alpha^{-1} \phi_{t}^{A}$ is clearly a desired differential. Uniqueness follows from Lemma 2.

LEMMA 5. Let $\phi, \psi$ be square integrable holomorphic semiexact differentials on $R$ and suppose that $\phi$ is exact on $R \backslash A$. Then

$$
(\phi, \psi)_{R}= \begin{cases}-2 i \int_{B} \phi \int_{A} \operatorname{Re} \psi & \text { if } \operatorname{Re} \psi \text { is distinguished } \\ -2 \int_{B} \phi \int_{A} \operatorname{Im} \psi & \text { if } \operatorname{Im} \psi \text { is distinguished }\end{cases}
$$

where $(\phi, \psi)_{R}:=\iint_{R} \phi \wedge \bar{\psi}^{*}$.

There seem to be no explicit references to Lemma 5. But we can prove it easily by noting that

$$
(\phi, \psi)_{R}=(\phi, \psi-\bar{\psi}+\bar{\psi})_{R}=(\phi, 2 i \operatorname{Im} \psi)_{R}
$$


and

$$
(\phi, \psi)_{R}=(\phi, \psi+\bar{\psi}-\bar{\psi})_{R}=(\phi, 2 \operatorname{Re} \psi)_{R}
$$

Each lemma in this section can be easily rephrased in terms of S-functions.

\section{Extremal slit tori.}

3.1. In this section we shall prove the following theorem.

THEOREM 2. Let $\left[T_{\nu},\left\{A_{\nu}, B_{\nu}\right\}, i_{\nu}\right]$ be the hydrodynamic continuations of $(R,\{A, B\})$ with respect to $d \Phi_{\nu}$ and set $\tau_{\nu}=\tau\left[T_{\nu},\left\{A_{\nu}, B_{\nu}\right\}, i_{\nu}\right], \nu=0,1$. Then

$$
\operatorname{Im} \tau_{0} \leq \operatorname{Im} \tau\left[T,\left\{A_{T}, B_{T}\right\}, i\right] \leq \operatorname{Im} \tau_{1}
$$

for every $\left[T,\left\{A_{T}, B_{T}\right\}, i\right] \in C(R,\{A, B\})$.

$\left(\mathrm{I}^{\prime}\right) \operatorname{Im} \tau\left[T,\left\{A_{T}, B_{T}\right\}, i\right]=\operatorname{Im} \tau_{\nu}$ holds if and only if

$$
\left[T,\left\{A_{T}, B_{T}\right\}, i\right]=\left[T_{\nu},\left\{A_{\nu}, B_{\nu}\right\}, i_{\nu}\right], \quad \nu=0,1 .
$$

(II) Every component of $T_{0} \backslash i_{0}(R)$ (resp. $\left.T_{1} \backslash i_{1}(R)\right)$ is a closed arc which is geodesic parallel (resp. geodesic orthogonal) to $A_{0}$ (resp. $\left.A_{1}\right)$.

(III) The area of $T_{\nu} \backslash i_{\nu}(R)$ vanishes for $\nu=0,1$.

PROOF. Take an arbitrary compact continuation $\left[T,\left\{A_{T}, B_{T}\right\}, i\right]$ of $(R,\{A, B\})$ and consider the normal holomorphic differential $\phi^{T}$ on $T$ with respect to $\left\{A_{T}, B_{T}\right\}$. The pull-back $\phi:=i^{*}\left(\phi^{T}\right)$ of $\phi^{T}$ is a holomorphic semiexact differential on $R$, which is evidently square integrable. Furthermore,

$$
\int_{A} \phi=1 \quad \text { and } \quad \int_{B} \phi=\tau\left[T,\left\{A_{T}, B_{T}\right\}, i\right] .
$$

Set $\phi_{0}=d \Phi_{0}$. Then, applying Lemma 5 to $\phi_{0}-\phi$ and $\phi_{0}$, we have

$$
\left(\phi_{0}-\phi, \phi_{0}\right)_{R}=-2 \int_{B}\left(\phi_{0}-\phi\right) \int_{A} \operatorname{Im} \phi_{0}=0 .
$$

Hence, we have

$$
0 \leq\left\|\phi_{0}-\phi\right\|_{R}^{2}=\|\phi\|_{R}^{2}-\left\|\phi_{0}\right\|_{R}^{2} .
$$

Since $\|\phi\|_{R}^{2}=\left\|\phi^{T}\right\|_{i(R)}^{2} \leq\left\|\phi^{T}\right\|_{T}^{2}=2 \operatorname{Im} \tau\left[T,\left\{A_{T}, B_{T}\right\}, i\right]$ and $\left\|\phi_{0}\right\|_{R}^{2}=2 \operatorname{Im} \tau_{0}$, we have

$$
\operatorname{Im} \tau_{0} \leq \operatorname{Im} \tau\left[T,\left\{A_{T}, B_{T}\right\}, i\right] .
$$

Equality holds if and only if $\phi=\phi_{0}$, which means

$$
\left[T,\left\{A_{T}, B_{T}\right\}, i\right]=\left[T_{0},\left\{A_{0}, B_{0}\right\}, i_{0}\right] .
$$

Thus we have proved that $\left[T_{0},\left\{A_{0}, B_{0}\right\}, i_{0}\right]$ is the unique solution to the problem of minimizing $\operatorname{Im} \tau$ in $C(R,\{A, B\})$. We have also proved that $T_{0} \backslash i_{0}(R)$ is of zero area (with respect to the metric $\left.\left|\phi^{T_{0}}\right|\right)$.

Each component of $T_{0} \backslash i_{0}(R)$ is, by Theorems 1 and $1^{\prime}$, on a (horizontal) trajectory of the quadratic differential $\left(\phi^{T_{0}}\right)^{2}$. This means that each component is an arc which is geodesic parallel to $A_{0}$. Some arcs may reduce to single points.

Thus we have proved the theorem for $\nu=0$. The other extremal problem $(\nu=1)$ can be dealt with in the same manner.

Similar results can be proved for any hydrodynamic continuation. See $§ 5.2$. 
3.2. We have also proved the following

TheOREM $2^{\prime}$. Define $\Sigma: C(R,\{A, B\}) \rightarrow \mathbf{R}$ by

$$
\Sigma\left[T,\left\{A_{T}, B_{T}\right\}, i\right]=\left\|\phi^{T}\right\|_{i(R)},
$$

where $\left[T,\left\{A_{T}, B_{T}\right\}, i\right] \in C(R,\{A, B\})$ and $\phi^{T}$ is the normal differential of the first kind on $T$ with respect to $\left\{A_{T}, B_{T}\right\}$. Then $\Sigma$ has its minimum (resp. maximum) for $\left[T_{0},\left\{A_{0}, B_{0}\right\}, i_{0}\right]$ (resp. $\left.\left[T_{1},\left\{A_{1}, B_{1}\right\}, i_{1}\right]\right)$. The minimum (resp. maximum) value is attained by $\left[T_{0},\left\{A_{0}, B_{0}\right\}, i_{0}\right]$ (resp. $\left.\left[T_{1},\left\{A_{1}, B_{1}\right\}, i_{1}\right]\right)$ only.

Theorems 2 and $2^{\prime}$ are analogous to the well-known theorems for extremal slit annuli. See Carleman [4], Reich-Warschawski [17], Rengel [18], Sakai [23] and Thao [30]; cf. [21 and 22], too. Our theorems also have a close connection with Grötzsch's differential geometric approach [7, 8]. Cf. Jenkins [13], too.

The importance of Theorems 2 and $2^{\prime}$ lies not only in such an analogy to classical results as above, but also in the useful applications to the theory of continuations of an open Riemann surface. See, e.g., the proof of Proposition 1 below.

4. Moduli of hydrodynamic continuations. To proceed further, we have to compute the $B$-period $\tau_{t}$ of $\phi_{t}$ in Lemma $4,-1<t \leq 1$. Let $\phi_{0}^{A}, \phi_{0}^{B}$ be the same as in Lemma 1. We first prove the following

Proposition 1. $\rho(R):=\left(a_{0}^{\prime} b_{0}^{\prime \prime}-a_{0}^{\prime \prime} b_{0}^{\prime}-1\right) / 2 a_{0}^{\prime}$ is nonnegative.

ProOF. Observe first that $\left(1+i b_{0}^{\prime}\right)^{-1} \phi_{0}^{B}$ is identical with

$$
\phi_{s}, \quad s=(2 / \pi) \tan ^{-1}\left(1 / b_{0}^{\prime}\right) \in(-1,1]
$$

so that we have

$$
\operatorname{Im} \tau_{s}=\operatorname{Im}\left[i b_{0}^{\prime \prime} /\left(1+i b_{0}^{\prime}\right)\right]=b_{0}^{\prime \prime} /\left(1+b_{0}^{\prime 2}\right)
$$

Since

$$
\operatorname{Im} \tau_{0}=\operatorname{Im}\left[\left(a_{0}^{\prime \prime}+i\right) / a_{0}^{\prime}\right]=1 / a_{0}^{\prime},
$$

we have by Theorem 2

$$
1 / a_{0}^{\prime} \leq b_{0}^{\prime \prime} /\left(1+b_{0}^{\prime 2}\right) .
$$

Because $b_{0}^{\prime}=a_{0}^{\prime \prime}$ by Lemma 3 , it follows immediately that

$$
\rho(R)=\left(a_{0}^{\prime} b_{0}^{\prime \prime}-a_{0}^{\prime \prime} b_{0}^{\prime}-1\right) / 2 a_{0}^{\prime}
$$

is nonnegative.

The differentials $\phi_{0}^{A}$ and $\phi_{0}^{B}$ form a basis (over reals) for the space of holomorphic differentials on $R$ with distinguished real parts (cf. [25]). Therefore there are two real numbers $\xi, \eta$ such that

$$
i e^{-\frac{1}{2} \pi i t} \phi_{t}=\xi \phi_{0}^{A}+\eta \phi_{0}^{B}
$$

Comparing the $A$-period of both sides, we have

$$
\xi=\left(\cos \frac{1}{2} \pi t-b_{0}^{\prime} \sin \frac{1}{2} \pi t\right) / a_{0}^{\prime}, \quad \eta=\sin \frac{1}{2} \pi t .
$$

Hence, the $B$-period $\tau_{t}$ of $\phi_{t}$ is equal to

$$
\begin{gathered}
-i e^{\frac{1}{2} \pi i t}\left\{a_{0}^{\prime^{-1}}\left(-1+i a_{0}^{\prime \prime}\right)\left(\cos \frac{1}{2} \pi t-b_{0}^{\prime} \sin \frac{1}{2} \pi t\right)+i b_{0}^{\prime} \sin \frac{1}{2} \pi t\right\} \\
=\left\{a_{0}^{\prime \prime} / a_{0}^{\prime}+i / a_{0}^{\prime}\right\}+i \rho(R)+\rho(R) \exp \left[\left(t-\frac{1}{2}\right) \pi i\right] .
\end{gathered}
$$

Since $\rho(R)$ is nonnegative by Proposition 1 , we know that

$$
\tau_{t}=\left\{\tau_{0}+i \rho(R)\right\}+\rho(R) \exp \left[\left(t-\frac{1}{2}\right) \pi i\right]
$$

moves on the full circle $K$ of radius $\rho(R)$ with the center at $\tau^{*}:=\tau_{0}+i \rho(R)$, when 
$t$ ranges over $(-1,1]$. We have hence proved

THEOREM 3. Let $\Phi_{t}$ be the regular $S_{t}$-function on $R$ with $\int_{A} d \Phi_{t}=1, \tau_{t}$ the modulus of the hydrodynamic continuation of $(R,\{A, B\})$ with respect to $d \Phi_{t},-1<$ $t \leq 1$. Then the set $\left\{\tau_{t} \mid-1<t \leq 1\right\}$ coincides with the circle $K:\left\{\left|\tau-\tau^{*}\right|=\rho(R)\right\}$, where

$$
\rho(R)=\left(a_{0}^{\prime} b_{0}^{\prime \prime}-a_{0}^{\prime \prime} b_{0}^{\prime}-1\right) / 2 a_{0}^{\prime} \quad(\geq 0)
$$

and $\tau^{*}=\tau_{0}+i \rho(R)$. More precisely, $t$ parametrizes $K$ by

$$
\tau_{t}=\tau^{*}+\rho(R) \exp \left[\left(t-\frac{1}{2}\right) \pi i\right], \quad-1<t \leq 1 .
$$

COROllary. (1) $\operatorname{Re} \tau_{1}=\operatorname{Re} \tau_{0}$.

(2) $\rho(R)=\frac{1}{2 i}\left(\tau_{1}-\tau_{0}\right)$.

(3) $\tau^{*}=\frac{1}{2}\left(\tau_{1}+\tau_{0}\right)$.

\section{Extremal property of hydrodynamic continuations.}

5.1. Now we shall sharpen Theorems 2 and 3 as follows.

THEOREM 4. The set $M(R,\{A, B\})$ is contained in the disk $\left\{\left|\tau-\tau^{*}\right| \leq \rho(R)\right\}$. Furthermore, $\tau \in M(R,\{A, B\})$ satisfies $\left|\tau-\tau^{*}\right|=\rho(R)$ if and only if it is the modulus of a hydrodynamic continuation of $(R,\{A, B\})$.

ProOF. Let $\left[T,\left\{A_{T}, B_{T}\right\}, i\right]$ be an arbitrary point of $C(R,\{A, B\})$ and $\tau$ its modulus. Let $\phi^{T}$ be the normal differential of the first kind on $\left(T,\left\{A_{T}, B_{T}\right\}\right), \phi:=$ $i^{*}\left(\phi^{T}\right)$ its pull-back to $R$ via $i$. Then

$$
\tau=\int_{B_{T}} \phi^{T}=\int_{B} \phi .
$$

Observe that, for every $t \in(-1,1]$,

$$
\int_{A} e^{-\frac{1}{2} \pi i t}\left(\phi_{t}-\phi\right)=0,
$$

and $\operatorname{Im}\left[e^{-\frac{1}{2} \pi i t} \phi_{t}\right]$ is distinguished. Hence we can apply Lemma 5 and obtain

$$
\begin{aligned}
\left(\phi_{t}-\phi, \phi_{t}\right)_{R} & =\left(e^{-\frac{1}{2} \pi i t}\left(\phi_{t}-\phi\right), e^{-\frac{1}{2} \pi i t} \phi_{t}\right)_{R} \\
& =-2 \int_{B} e^{-\frac{1}{2} \pi i t}\left(\phi_{t}-\phi\right) \cdot \int_{A} \operatorname{Im}\left[e^{-\frac{1}{2} \pi i t} \phi_{t}\right] \\
& =2 e^{-\frac{1}{2} \pi i t}\left(\tau_{t}-\tau\right) \sin \frac{1}{2} \pi t
\end{aligned}
$$

It follows by simple computations that

$$
\begin{aligned}
0 & \leq\left\|\phi_{t}-\phi\right\|_{R}^{2}=2 \operatorname{Re}\left(\phi_{t}-\phi, \phi_{t}\right)_{R}+\|\phi\|_{R}^{2}-\left\|\phi_{t}\right\|_{R}^{2} \\
& \leq-2 \operatorname{Im}\left[e^{-\pi i t}\left(\tau_{t}-\tau\right)\right] .
\end{aligned}
$$

Since $\tau_{t}=\tau^{*}+\rho(R) \exp \left[\left(t-\frac{1}{2}\right) \pi i\right]$ by Theorem 3 , we have $\operatorname{Im}\left[e^{-\pi i t}\left(\tau^{*}-\tau\right)\right] \leq$ $\rho(R)$. This inequality holds for every $t \in(-1,1]$, so that $\left|\tau-\tau^{*}\right| \leq \rho(R)$. Thus we have proved the first assertion.

As for the second assertion we only need to prove the only if part, since the if part was established in Theorem 3. Let $\left[T,\left\{A_{T}, B_{T}\right\}, i\right] \in C(R,\{A, B\})$ and assume $\tau:=\tau\left[T,\left\{A_{T}, B_{T}\right\}, i\right]$ satisfies $\left|\tau-\tau^{*}\right|=\rho(R)$. Then $\tau=\tau_{t}$ for some $t \in(-1,1]$. It follows immediately from inequality $(*)$ that the pull-back of the normal holo- 
morphic differential on $\left(T,\left\{A_{T}, B_{T}\right\}\right)$ is identical with $\phi_{t}$. Hence $\left[T,\left\{A_{T}, B_{T}\right\}, i\right]$ is the hydrodynamic continuation with respect to $\phi_{t}$.

5.2. From Theorems 3 and 4 we can conclude that hydrodynamic continuations have extremal properties (cf. Theorem 2 too). Consider, for instance, the extremal problem to maximize $\operatorname{Im} \frac{1}{\tau}$ in $C(R,\{A, B\})$. By Theorems 3 and 4 we know that there is a unique solution. The solution is given by the hydrodynamic continuation $\left[T^{\#},\left\{A^{\#}, B^{\#}\right\}, i^{\#}\right]$ such that each component of $T^{\#} \backslash i^{\#}(R)$ is a slit which is geodesic parallel to $B^{\#}$ ( $B^{\#}$ is assumed, as usual, to be geodesic). This extremal problem is dealt with by Grötzsch. See [6]. By computations-or by simple geometric observation-we see that the coordinate origin, $\tau_{0}$, and $\tau^{\#}$ are collinear, $\tau^{\#}$ being the modulus of the solution.

Let $\tau_{\#}$ be a complex number with $\operatorname{Im} \tau_{\#}>0$ and assume (i) the coordinate origin, $\tau_{\#}$, and $\tau_{1}$ are collinear, and (ii) $\left|\tau_{\#}-\tau^{*}\right|=\rho(R)$. Then the hydrodynamic continuation $\left[T_{\#},\left\{A_{\#}, B_{\#}\right\}, i_{\#}\right]$ with modulus $\tau_{\#}$ minimizes $\operatorname{Im} \frac{1}{\tau}$ in $C(R,\{A, B\})$ and each component of $T_{\#} \backslash i_{\#}(R)$ is a slit which is geodesic orthogonal to $B_{\#}$.

6. The complete description of the moduli set $M(R,\{A, B\})$.

6.1. We shall now show that every point in the disk $\left\{\left|\tau-\tau^{*}\right| \leq \rho(R)\right\}$ is obtained by some $\left[T,\left\{A_{T}, B_{T}\right\}, i\right] \in C(R,\{A, B\})$. To this end we begin with a special case where $R$ is the interior of a compact bordered Riemann surface $\bar{R}$. Let $\partial R$ denote the border of $\bar{R}$ and let $\beta_{1}, \beta_{2}, \ldots, \beta_{N}$ be the contours with $\partial R=\beta_{1}+\beta_{2}+\cdots+\beta_{N}$. Let $h, k$ be two positive numbers with $h+k=1$ and set $\Phi_{h k}=h \Phi_{0}+k \Phi_{1}, \phi_{h k}=$ $d \Phi_{h k}$.

Note that $\Phi_{0}, \Phi_{1}$ and $\Phi_{h k}$ are single-valued near $\partial R$. Their boundary values are determined modulo additive constants. In other words, the image curve $\Phi_{h k}\left(\beta_{i}\right)$ is determined only up to euclidean translations. Since the convexity of a plane curve is preserved under euclidean motions, it makes sense to speak of the convexity of $\Phi_{h k}\left(\beta_{i}\right)$.

LEMMA 6. Let $t \in(-1,1]$ and $h, k>0, h+k=1$. Set

$$
r=\left\{h^{2} \cos ^{2} \frac{1}{2} \pi t+k^{2} \sin ^{2} \frac{1}{2} \pi t\right\}
$$

and

$$
s=(2 / \pi) \tan ^{-1}\left((k / h) \tan \frac{1}{2} \pi t\right), \quad-1<s \leq 1 .
$$

Then

(1) $h \cos \frac{1}{2} \pi t \phi_{0}+i k \sin \frac{1}{2} \pi t \phi_{1}=\operatorname{Re}^{\frac{1}{2} \pi i s} \phi_{-s}$, and

(2) $\operatorname{Re}\left[e^{\frac{1}{2} \pi i t} \Phi_{h k}\right]=r \operatorname{Re}\left[e^{\frac{1}{2} \pi i s} \Phi_{-s}\right]+$ const. on $\beta_{i}(1 \leq i \leq N)$, $\Phi_{-s}$ being an (arbitrarily fixed) integral of $\phi_{-s}$.

PROOF. To prove (1) we set $\tilde{\phi}:=h \cos \frac{1}{2} \pi t \phi_{0}+i k \sin \frac{1}{2} \pi t \phi_{1}$. Then we know $\operatorname{Im} \tilde{\phi}=0$ along $\partial R$ and furthermore $\int_{A} \tilde{\phi}=e^{\frac{1}{2} \pi i s}$. These two conditions characterize the differential $\operatorname{Re}^{\frac{1}{2} \pi i s} \phi_{-s}$ (see Lemma 4), so that assertion (1) follows.

Assertion (2) is an easy consequence of $(1)$, for we have $\operatorname{Re}\left[e^{\frac{1}{2} \pi i t} \phi_{h k}\right]=\operatorname{Re} \tilde{\phi}$ on $\partial R$.

From this lemma we can conclude, as in [2] (see p. $181 \mathrm{ff}$.), that the equation $\operatorname{Re}\left[e^{\frac{1}{2} \pi i t} \Phi_{h k}\right]=b$ has at most two solutions on $\beta_{i}$ for each $b \in \mathbf{R}$ and $i=1,2, \ldots, N$. This means that each $\Phi_{h k}\left(\beta_{i}\right)$ is a convex (analytic) curve. 
6.2. We shall next show the following lemma.

LEMMA 7. Let $h, k>0, h+k=1$. Then

$$
\int_{\beta_{i}} d \arg \phi_{h k}=-1, \quad 1 \leq i \leq N .
$$

ProOF. Set $F=\phi_{1} / \phi_{0}$. Then $F$ is a single-valued (nonconstant) meromorphic function on $\bar{R}$, which is holomorphic on $R$. Applying the argument principle to $F$, we see that $F \mid R$, the restriction of $F$ onto $R$, takes on no purely imaginary values. Hence, either $\operatorname{Re} F>0$ throughout $R$ or else $\operatorname{Re} F<0$ throughout $R$.

We may assume that $A$ is so chosen that $i_{0}(A)$ is a geodesic with respect to the metric induced by the transplant of $\phi_{0}$ by $i_{0}^{-1}$. Then we have

$$
1=\int_{A} \phi_{1}=\int_{A} F \phi_{0}=\int_{A}(\operatorname{Re} F) \phi_{0} .
$$

This shows that $\operatorname{Re} F>0$ throughout $R$. Consequently

$$
\int_{\beta_{i}} d \arg \phi_{h k}=\int_{\beta_{i}} d \arg \phi_{0}=-1 .
$$

By Lemmas 6 and 7 we have

Proposition 2. Let $h, k>0, h+k=1$, and let $R$ be the interior of a compact bordered Riemann surface of genus one. Then each contour of $R$ is mapped by $\Phi_{h k}=h \Phi_{0}+k \Phi_{1}$ onto a negatively oriented convex analytic curve.

By considering each contour separately, we obtain a torus $T_{h k}$, a naturally defined conformal embedding $i_{h k}: R \rightarrow T_{h k}$ such that the transplant of $\phi_{h k}$ by $i_{h k}^{-1}$ extends to a holomorphic differential $\psi_{h k}$ on $T_{h k}$. Since the marking $\{A, B\}$ of $R$ is easily transferred to a marking $\left\{A_{h k}, B_{h k}\right\}$ of $T_{h k}$ via $i_{h k}$, we have obtained a marked realization $\left(T_{h k},\left\{A_{h k}, B_{h k}\right\}, i_{h k}\right)$ of $(R,\{A, B\})$. Furthermore, $\tau\left[T_{h k},\left\{A_{h k}, B_{h k}\right\}, i_{h k}\right]=h \tau_{0}+k \tau_{1}$, for we have

$$
\int_{A_{h k}} \psi_{h k}=\int_{A} \phi_{h k}=1 \quad \text { and } \quad \int_{B_{h k}} \psi_{h k}=\int_{B} \phi_{h k}=h \tau_{0}+k \tau_{1} .
$$

It follows that any point on the vertical diameter of the disk $\left\{\left|\tau-\tau^{*}\right| \leq \rho(R)\right\}$ is the modulus of a compact continuation of $(R,\{A, B\})$.

The same reasoning as above clearly applies to any pair $\phi_{t}$ and $\phi_{t+1},-1<t<0$. Hence we have proved

PROPOSITION 3. If $R$ is the interior of a compact bordered Riemann surface of genus one, $M(R,\{A, B\})$ is a closed disk in the upper half plane.

6.3. Let $\Psi_{h k}$ be any (but fixed) integral of $\psi_{h k}$. We know (see, e.g., Siegel [28, p. $50 \mathrm{ff}]$.$) that \Psi_{h k}$ is a single-valued univalent holomorphic function on the simply connected surface $T_{h k} \backslash\left(A_{h k} \cup B_{h k}\right)$ which is obtained by cutting $T_{h k}$ along $A_{h k}$ and $B_{h k}$. Hence $\Phi_{h k}$ is a single-valued univalent holomorphic function on $\bar{R} \backslash(A \cup B)$, so that $\Phi_{h k}\left(\beta_{i}\right), i=1,2, \ldots, N$, are mutually disjoint convex curves and any one of them lies outside the others. 
Each component of $T_{h k} \backslash i_{h k}(R)$ is a convex set. Any marked realization equivalent to $\left(T_{h k},\left\{A_{h k}, B_{h k}\right\}, i_{h k}\right)$ evidently has the same property. So, $\left[T_{h k},\left\{A_{h k}, B_{h k}\right\}\right.$, $\left.i_{h k}\right]$ may be called a compact continuation with convex complements. The proof of Proposition 3 actually shows that every interior point of the disk $\left\{\left|\tau-\tau^{*}\right| \leq \rho(R)\right\}$ is the modulus of a compact continuation with convex complements.

6.4. To pass to the general open case $(R,\{A, B\})$, we take an exhaustion $\left\{R_{n}\right\}_{n=1}^{\infty}$ of $R$ by regular regions of genus one. We may assume $R_{1} \supset A \cup B$. Let $\phi_{t}^{(n)}=$ $d \Phi_{t}^{(n)}$ be the holomorphic differentials on $R_{n}$ such that $\Phi_{t}^{(n)}$ are $\mathrm{S}_{t}$-functions and $\int_{A} \phi_{t}^{(n)}=1$. For $h, k>0, h+k=1$, we set $\Phi_{h k}^{(n)}=h \Phi_{0}^{(n)}+k \Phi_{1}^{(n)}, \phi_{h k}^{(n)}=d \Phi_{h k}^{(n)}$. It is known that $\left\{\phi_{h k}^{(n)}\right\}_{n=1}^{\infty}$ converges to $\phi_{h k}$ locally uniformly on $R$.

We cut $R$ along $A, B$ and denote by $\tilde{R}$ the resulting surface, that is, $\tilde{R}=$ $R \backslash(A \cup B)$. If we set $\tilde{R}_{n}=\tilde{R} \cap R_{n}$, then $\tilde{R}=\bigcup_{n=1}^{\infty} \tilde{R}_{n}$. As was noted earlier, $\Phi_{h k}^{(n)}$ are single-valued univalent holomorphic functions on $\tilde{R}_{n}$. We may assume that $\left\{\Phi_{h k}^{(n)}\right\}_{n=1}^{\infty}$ converges to $\Phi_{h k}$ locally uniformly on $\tilde{R}$. Then, by a theorem of Hurwitz, $\Phi_{h k}$ is univalent on $\tilde{R}$. In particular, $\Phi_{h k}$ gives rise to a compact continuation of $(R,\{A, B\})$, whose modulus is clearly $h \tau_{0}+k \tau_{1}$. We can now repeat the argument preceding Proposition 3 and see that every point in the disk $\left\{\left|\tau-\tau^{*}\right| \leq \rho(R)\right\}$ is the modulus of a compact continuation of $(R,\{A, B\})$.

Combining these results together with those in $\S 5$, we have the following refinement of Heins' theorem (cf. [9]).

THEOREM 5. For any marked open Riemann surface $(R,\{A, B\})$ of genus one $M(R,\{A, B\})$ is a closed disk in the upper half plane. Furthermore, $\tau \in$ $M(R,\{A, B\})$ is the modulus of a hydrodynamic continuation of $(R,\{A, B\})$ if and only if $\tau$ is a boundary point of $M(R,\{A, B\})$. This correspondence between $\partial M(R,\{A, B\})$ and the space of hydrodynamic continuations is one-to-one.

7. Nonuniqueness. We have already shown that every boundary point of $M(R,\{A, B\})$ arises from a unique element in $C(R,\{A, B\})$, which is actually a hydrodynamic continuation of $(R,\{A, B\})$. As for the interior points of $M(R\{A, B\})$, this is not the case in general. Namely we have

Proposition 4. There exists a marked open Riemann surface of genus one which has (at least) two distinct compact continuations with the same modulus.

Proof. Take an arbitrary torus $T$ with a canonical homology basis $\{A, B\}$. Let $D$ be a nonconvex closed connected set of positive area on $T$ such that $D \cap A=$ $D \cap B=\varnothing$. Remove $D$ from $T$ and denote by $R$ the resulting open Riemann surface of genus one. By Theorem 5 we know that $M(R\{A, B\})$ is a closed disk of positive radius and $[T,\{A, B\}, i]$ corresponds to an interior point $\tau$ of $M(R,\{A, B\})$, where $i$ is the restriction of the identity mapping (of $T$ ) to $R$.

On the other hand, the same point $\tau$ is also realized by a compact continuation $\left[T^{\prime},\left\{A^{\prime}, B^{\prime}\right\}, i^{\prime}\right]$ of $(R,\{A, B\})$ such that $T^{\prime} \backslash i^{\prime}(R)$ is a convex set. See $\S 6.3$. It is easily seen that $[T,\{A, B\}, i] \neq\left[T^{\prime},\left\{A^{\prime}, B^{\prime}\right\}, i^{\prime}\right]$ since any conformal mapping of a torus onto another preserves the convexity of subsets.

Similar problems to ours are discussed by Ioffe. See [11 and 12]. He also considers the case of higher genera; however, he always restricts himself to the compact bordered surfaces. 


\section{Span.}

8.1. The radius $\rho(R)$ of the disk $M(R,\{A, B\})$ represents the size of the ideal boundary of $R$. Indeed, we have the following

THEOREM 6. $\rho(R)=0$ if and only if $R \in O_{A D}$.

This theorem immediately follows from Theorem 5 and the well-known uniqueness theorem which asserts $R \in O_{A D}$ if and only if $C(R,\{A, B\})$ consists of a single point. See Mori [14] and Oikawa [16]. Cf. also [1, 2, 21, and 22].

8.2. Let $\left\{R_{n}\right\}_{n=1}^{\infty}$ be the same as in $\S 6.4$. Then Cauchy's theorem yields

$$
\begin{aligned}
0 & =\int_{\partial R_{n}} \Phi_{1} \phi_{0}+\left(\int_{A} \phi_{1} \int_{B} \phi_{0}-\int_{B} \phi_{1} \int_{A} \phi_{0}\right) \\
& =\int_{\partial R_{n}} \Phi_{1} \phi_{0}+\left(\tau_{0}-\tau_{1}\right),
\end{aligned}
$$

$n=1,2, \ldots$ Hence, by the corollary to Theorem 3 , we have

THEOREM 7 .

$$
\frac{\rho(R)}{\pi}=\operatorname{Re}\left\{\frac{1}{2 \pi i} \int_{\partial R} \Phi_{1} \phi_{0}\right\}=\lim _{n \rightarrow \infty} \operatorname{Re}\left\{\frac{1}{2 \pi i} \int_{\partial R_{n}} \Phi_{1} \phi_{0}\right\} .
$$

This theorem shows an analogue between $\sigma(R):=\rho(R) / \pi$ and Schiffer's span for planar regions [24]. To see this, let us recall the (usual) definition of the span $s(G, \zeta)$ of a plane region $G$ with respect to the reference point $\zeta$ in $G$. Suppose $f_{0}$ (resp. $f_{1}$ ) is the normalized horizontal (resp. vertical) slit mapping of $G$ with respect to $\zeta$. Namely, $f_{0}(\zeta)=f_{1}(\zeta)=\infty$ and their Laurent expansions about $\zeta$ shall be of the form $1 /(z-\varsigma)+a_{k}(z-\varsigma)+a_{k}^{\prime}(z-\varsigma)^{2}+\cdots, k=0,1$. Then, $s(G, \varsigma)$ is defined as $a_{0}-a_{1}[\mathbf{2 4}]$, which is known to be real. Next, we observe that $s(G, \varsigma)$ is also represented as

$$
\operatorname{Re}\left\{\frac{1}{2 \pi i} \int_{\partial G} f_{1} d f_{0}\right\}=\lim _{n \rightarrow \infty} \operatorname{Re}\left\{\frac{1}{2 \pi i} \int_{\partial G_{n}} f_{1} d f_{0}\right\},
$$

where $\left\{G_{n}\right\}_{n=1}^{\infty}$ is a regular exhaustion of $G$. (This fact is, though a simple consequence of Cauchy's theorem, scarcely found in the literature.) Thus we have seen that $\sigma(R)$ and $s(G, \zeta)$ have similar expressions.

Other generalizations of Schiffer's span are also known; see Rodin-Sario [21] and Sario-Oikawa [22], for example. However, they are closely related to the degeneration of $H D$ - or $K D$-functions rather than $A D$-functions. Also they usually depend on the reference point used and the choice of local parameter about that point, while our span $\sigma(R)$ needs no reference point.

\section{REFERENCES}

1. L. V. Ahlfors and A. Beurling, Conformal invariants and function-theoretic null-sets, Acta Math. 83 (1950), 101-129.

2. L. V. Ahlfors and L. Sario, Riemann surfaces, Princeton Univ. Press, Princeton, N.J., 1960, $382 \mathrm{pp}$.

3. S. Bochner, Fortsetzung Riemannscher Flächen, Math. Ann. 98 (1927), 406-421.

4. T. Carleman, Über ein Minimalproblem der mathematischen Physik, Math. Z. 1 (1918), $208-212$.

5. H. M. Farkas and I. Kra, Riemann surfaces, Springer, New York and Berlin, 1980, 337 pp. 
6. H. Grötzsch, Die Werte des Doppelverhältnisses bei schlichter konformer Abbildung, Sitzungsber. Preuss. Akad. Wiss. Berlin (1933), 501-515.

7. __ , Über Flächensätze der konformen Abbildung, Jber. Deutsch. Math.-Verein. 44 (1934), 266269.

8. __ Einige Bemerkungen zur schlichten konformen Abbildung, Jber. Deutsch. Math.-Verein. 44 (1934), 270-275.

9. M. Heins, A problem concerning the continuation of Riemann surfaces, Contributions to the Theory of Riemann Surfaces (edited by L. V. Ahlfors et al.), Princeton Univ. Press, Princeton, N.J., 1953, pp. 55-62.

10. A. Hurwitz and R. Courant, Vorlesungen über allgemeine Funktionentheorie und elliptische Funktionen. Geometrische Funktionentheorie, Vierte Aufl. (mit einem Anhang von H. Röhrl), Springer, Berlin and New York, 1964, 706 pp.

11. M. S. Ioffe, Conformal and quasi-conformal imbedding of one finite Riemann surface into another, Soviet Math. Dokl. 13 (1972), 75-78.

12. _ On a problem of the variational calculus in the large for conformal and quasi-conformal mappings of one finite Riemann surface in another, Soviet Math. Dokl. 14 (1973), 1576-1579.

13. J. A. Jenkins, Univalent functions and conformal mapping, Springer, Berlin, 1958, $169 \mathrm{pp}$.

14. A. Mori, A remark on the prolongation of Riemann surfaces of finite genus, J. Math. Soc. Japan 4 (1952), 27-30.

15. K. Oikawa, On the prolongation of an open Riemann surface of finite genus, Kōdai Math. Sem. Rep. 9 (1957), 34-41.

16. $\ldots$ On the uniqueness of the prolongation of an open Riemann surface of finite genus, Proc. Amer. Math. Soc. 11 (1960), 785-787.

17. E. Reich and S. Warschawski, On canonical conformal maps of regions of arbitrary connectivity, Pacific J. Math. 10 (1960), 965-985.

18. E. Rengel, Existenzbeweise für schlichte Abbildungen mehrfach zusammenhängender Bereiche auf geurisse Normalbereiche, Jber. Deutsch. Math.-Verein. 44 (1934), 51-55.

19. H. Renggli, Structural instability and extensions of Riemann surfaces, Duke Math. J. 42 (1975), 211-224.

20. I. Richards, On the classification of noncompact surfaces, Trans. Amer. Math. Soc. 106 (1963), 259-269.

21. B. Rodin and L. Sario, Principal functions (with an Appendix by M. Nakai), Van Nostrand, Princeton, N.J., 1968, 347 pp.

22. L. Sario and K. Oikawa, Capacity functions, Springer, Berlin and New York, 1969, $361 \mathrm{pp.}$

23. A. Sakai, On minimal slit domains, Proc. Japan Acad. 35 (1959), 128-133.

24. M. Schiffer, The span of multiply connected domains, Duke Math. J. 10 (1943), 209-216.

25. M. Shiba, On the Riemann-Roch theorem on open Riemann surfaces, J. Math. Kyoto Univ. 11 (1971), 495-525.

26. _ The Riemann-Huruitz relation, parallel slit covering map, and continuation of an open Riemann surface of finite genus, Hiroshima Math. J. 14 (1984), 371-399.

27. M. Shiba and K. Shibata, Hydrodymamic continuations of an open Riemann surface of finite genus, Complex Variables Theory Appl. 8 (1986).

28. C. L. Siegel, Topics in complex function theory, Vol. I, Wiley-Interscience, New York, 1969, $186 \mathrm{pp}$.

29. G. Springer, Introduction to Riemann surfaces, Addison-Wesley, Reading, Mass., 1957, 309 pp; Reprint: Chelsea, New York, 1981.

30. V. D. Thao, Über einige Flächeninhaltsformeln bei schlicht-konformer Abbildung von Kreisbogenschlitzgebieten, Math. Nachr. 74 (1976), 253-261.

31. S. Timmann, Einbettungen endlicher Riemannscher Flächen, Math. Ann. 217 (1975), 81-85.

Department of Mathematics, Hiroshima University, Hiroshima, Japan 